\title{
X-ray Irradiation activates immune response in human T-lymphocytes by eliciting a $\mathrm{Ca}^{2+}$ signaling cascade
}

\author{
Dominique Tandl ${ }^{1}$, Tim Sponagel ${ }^{1}$, Sebastian Fuck ${ }^{1}$, Timo Smit, ${ }^{3}$ Stephanie Hehlgans ${ }^{2}$, Burkhard Jakob ${ }^{3}$, \\ Claudia Fournier ${ }^{3}$, Franz Rödel ${ }^{2}$, Bastian Roth ${ }^{1}$, Anna Moroni $^{4}$, Gerhard Thiel $^{1(*)}$
}

\author{
Affiliations \\ ${ }^{1}$ Department of Biology, Technische Universität Darmstadt, Darmstadt, Germany \\ ${ }^{2}$ Department of Radiotherapy and Oncology, Goethe-University, Frankfurt am Main, Germany \\ ${ }^{3}$ Department of Biophysics, GSI Helmholtzzentrum für Schwerionenforschung, Darmstadt, Germany \\ ${ }^{4}$ Department of Biosciences and CNR IBF-Mi, Università degli Studi di Milano, Milano, Italy
}

Short title: $\mathrm{X}$-ray triggered $\mathrm{Ca}^{2+}$ signaling in $\mathrm{T}$ cells

\begin{abstract}
Radiation therapy is efficiently employed for eliminating cancer cells and reducing tumor growth. To further improving its therapeutic application it is mandatory to unravel the molecular effects of ionizing irradiation and to understand whether they support or counteract tumor therapy. Here we examine the impact of X-ray irradiation on immune activation of human $\mathrm{T}$ cells with single doses typically employed in tumor therapy. We discover that exposing cells to radiation triggers in a population of leukemic Jurkat $\mathrm{T}$ cells and in peripheral blood mononuclear cells (PBMCs) a canonical $\mathrm{Ca}^{2+}$ signaling cascade, which elicits immune activation of these cells. An early step in the signaling cascade is the initiation of sustained oscillations of the cytosolic $\mathrm{Ca}^{2+}$ concentration, an event mediated by store operated $\mathrm{Ca}^{2+}$ entry (SOCE) via an X-ray induced clustering of the Calcium Release-Activated Calcium Modulator 1 with the stromal interaction molecule 1 (Oari1/STIM1). A functional consequence of the $\mathrm{Ca}^{2+}$ signaling cascade is the translocation of the transcription factor nuclear factor of activated T cells (NFAT) from the cytosol into the nucleus where it elicits the expression of genes required for immune activation. These data imply that a direct activation of blood immune cells by ionizing irradiation has an impact on toxicity and therapeutic effects of radiation therapy.
\end{abstract}

\section{Introduction}

Ionizing radiation (IR) is a universal tool in medical diagnostics and therapy. At higher doses $(>1$ Gy) it serves as a major component in anti- tumor treatment (1). At lower doses ( $<1$ Gy) radiationtherapy is mainly used for treatment of a variety of inflammatory/degenerative and hyperproliferative benign diseases $(2,3)$. A major dose limitation of radiation therapy is posed by toxic effects to the surrounding heathy tissue. In that context, peripheral blood mononuclear cells (PBMCs) cover an interesting population of cells. They are sensitive to IR $(4,5)$ but, while cycling through the irradiated tissue, unavoidably exposed to it. Further, high dose exposure results in a suppression of immune functions (6). This includes among others killing of blood cells (7), induction of cell cycle arrests of immune cells (8) and triggering of pro-inflammatory processes $(9,10)$. By contrast, recent findings indicate an immune-stimulatory activity of high dose radiation with several studies describing a synergistic effect on local and distant tumor control when radiation therapy is combined with anti-checkpoint programmed death (PD-1) or its ligand PD-L1 immunotherapy $(11,12)$. Finally, recent data suggest that irradiation at lower doses display anti-inflammatory or immune modulatory effects with consequences on immune surveillance of non-cancerous cells (13).

In a previous study we have reported that X-rays irradiation with low to medium dose elicits cellular responses, which are typically associated with immune stimulation in naïve T-lymphocytes (14). These include an increase in cell diameter, up-regulation of CD25 membrane expression, interleukin-2 and interferon-gamma synthesis, an enhanced integrin-mediated adhesion of Jurkat cells, as model for T cells, or PBMCs to endothelial cells. Since many of these mechanisms are mediated by a $\mathrm{Ca}^{2+}$ signaling cascade we anticipate a causal interrelationship between radiation stress and induction of a $\mathrm{Ca}^{2+}$ signaling cascade (5, 
14). Here we aimed to examine whether clinically relevant doses of X-ray between 0.5 and $5 \mathrm{~Gy}$ trigger $\mathrm{Ca}^{2+}$ signaling events in $\mathrm{T}$ cells and whether these signal transduction cascades are relevant for immune stimulation. We observed that X-ray doses $<1$ Gy triggered in Jurkat cells long lasting episodes of $\mathrm{Ca}^{2+}$ oscillations with a delay of some 10 minutes. These oscillations are mediated by store operated $\mathrm{Ca}^{2+}$ entry (SOCE) via a radiation-induced clustering of Calcium Release-Activated Calcium Modulator 1 and the stromal interaction molecule 1 (Orail/STIM1) in the plasma membrane. Like immune stimulation by antigens also X-ray exposure induced $\mathrm{Ca}^{2+}$ entry via the SOCE pathway, which in turn stimulates the nuclear translocation of the transcription factor nuclear factor of activated T cells (NFAT). This stimulus-induced and $\mathrm{Ca}^{2+}$-dependent nuclear import is a well-known key step for cytokine production, proliferation and immune competence (15).

\section{Results}

\section{Ionizing irradiation elicits a delayed $\mathrm{Ca}^{2+}$ response in Jurkat cells}

It was previously shown that X-ray irradiation triggers an immune stimulation in $\mathrm{T}$ cells, which is mediated by an increase in the concentration of free $\mathrm{Ca}^{2+}$ in the cytoplasm $\left(\mathrm{Ca}^{2+}\right.$ cyt $)(14)$. To unravel a causal relationship between IR and $\mathrm{Ca}^{2+}$ cyt signaling cascades we monitored the immediate impact of $\mathrm{X}$-ray exposure on the level of this second messenger in Jurkat cells. Cells loaded with the fluorescent $\mathrm{Ca}^{2+}$ reporter dye Fluo4 were imaged in real-time on a fluorescence microscope directly coupled to an X-ray source. Fig. 1A shows that X-ray doses of 1 and $10 \mathrm{~Gy}$ did not elevate $\mathrm{Ca}^{2+}$ cyt levels within 10 min post irradiation. Combined with the finding that $\mathrm{Ca}^{2+}{ }_{\text {cyt }}$ in the same cells can be elevated by ionomycin (Fig. 1A) the data suggest that IR exposure has no immediate impact on $\mathrm{Ca}^{2+}{ }_{\text {cyt }}$ concentrations in Jurkat cells.

To capture potentially delayed $\mathrm{Ca}^{2+}$ cyt responses Fluo-4 fluorescence was monitored over an extended time window. Representative recordings in Fig. 1B indicate that most untreated cells maintained a constant low $\mathrm{Ca}^{2+}$ cyt over some hours of recording (Fig 1Bi). In 130 control cells we only observed in $20 \%$ of the cells some spontaneous and non-periodic excursions in $\mathrm{Ca}^{2+}$ cyt; the latter were mostly visible already at the start of the imaging (Fig. 1Bii). After exposure to 5 Gy approximately $50 \%$ of the cells exhibited characteristic delayed $\mathrm{Ca}^{2+}$ cyt oscillations. In the representative example in Fig.1Biii the cell started oscillating after a lag time of $65 \mathrm{~min}$ (Fig. 1B). Similar delayed and long lasting $\mathrm{Ca}^{2+}{ }_{\text {cyt }}$ oscillations were observed in a larger fraction of cells after irradiation with 5 Gy (Fig. 1C). The remaining cells either maintained a constant $\mathrm{Ca}^{2+}$ cyt or exhibited unspecific $\mathrm{Ca}^{2+}$ cyt excursions as in control cells (Fig. 1Bi,ii). For quantifying the probability of radiation-induced effects we consider here only $\mathrm{Ca}^{2+}$ oscillations, which occurred $\geq 10 \mathrm{~min}$ after start of the imaging. From this analysis it occurs that oscillations are only visible in ca. $10 \%$ of the control cells but in $56 \%$ of cells after irradiation with a dose of 5 Gy (Fig. S1A).

Experiments were repeated over a range of X-ray doses from 0.5 to $5 \mathrm{~Gy}$. These treatments also elicited with a high probability delayed $\mathrm{Ca}^{2+}$ cyt oscillations (Fig. S1A). The lag time between X-ray stimulation and onset of $\mathrm{Ca}^{2+}$ cyt signaling events varied considerable from one cell to the other. In $70 \%$ of the stimulated cells with $1 \mathrm{~Gy}$ the first detectable $\mathrm{Ca}^{2+}$ peak occurred between $10 \mathrm{~min}$ (fastest) and $72 \mathrm{~min}$ (slowest) after X- ray exposure. A plot of the average lag times as a function of X-ray dose indicates that this value is not significantly changing with the stimulation dose (Fig. 1D). A frequency analysis further reveals that $\mathrm{Ca}^{2+}$ cyt oscillates in response to $\mathrm{X}$-ray with 2 to $4 \mathrm{mHz}$. (Fig. 1E). This frequency remains largely constant over the range of irradiation doses.

The traces depicted in Fig. 1Biii, $\mathrm{C}$ show that the amplitude of the $\mathrm{Ca}^{2+}$ cyt excursions increases gradually with a saturation kinetics after onset of the oscillations. The peak values of the maximal $\mathrm{Ca}^{2+}{ }_{\text {cyt }}$ excursions are a function of the stimulation doses. From a fit of the data with a single saturating exponential function:

$$
\left.\mathrm{Y}=\mathrm{F}_{\max }\left(1-\mathrm{e}^{-\mathrm{x} / \mathrm{k}}\right)+\mathrm{F}_{0} \quad \text { (eqn. } 1\right)
$$

where $\mathrm{F}_{\max }$ is the maximal increase in peak fluorescence, $\mathrm{F}_{0}$ the background fluorescence of the control and $\mathrm{k}$ the dose for half maximal increase in fluorescence the half maximal $\mathrm{Ca}^{2+}$ cyt peak is achieved by $1.5 \mathrm{~Gy}$ (Fig. 1F).

In the case of antigen-mediated $\mathrm{T}$ cell activation, $\mathrm{Ca}^{2+}$ cyt oscillations are the result of store operated $\mathrm{Ca}^{2+}$ entry (SOCE), a mechanism, which can be imitated by thapsigargin (TG), an inhibitor for 
sarcoplasmic/endoplasmic reticulum calcium ATPase (SERCA). In Jurkat cells treated with $2 \mu \mathrm{M}$ TG the $\mathrm{Ca}^{2+}$ cyt signal increases to a new plateau; the latter, however, remains well below that of the maximal amplitude measured in response to X-ray stimulation. Notably, the maximal amplitude value was in the same range of those elicited by the $\mathrm{Ca}^{2+}$ ionophore ionomycin (Fig. 1F). This indicates that IR elicits $\mathrm{Ca}^{2+}$ cyt excursions to much higher levels than TG; the former amplitudes presumably exceeded the saturation of the Fluo-4 dye, which is about $1 \mu \mathrm{M}(16,17)$.

\section{$\mathrm{Ca}^{2+}$ cyt oscillations can be suppressed by buffering external $\mathrm{Ca}^{2+}$ and by blocking $\mathrm{Ca}^{2+}$ influx}

Stimulus-induced $\mathrm{Ca}^{2+}{ }_{\text {cyt }}$ oscillations can originate from a release of $\mathrm{Ca}^{2+}$ from internal stores or from entry via a plethora of $\mathrm{Ca}^{2+}$ permeable channels in the plasma membrane of $\mathrm{T}$ cells (18). To test the contribution of plasma membrane channels to this process, experiments similar to those in Fig. 1 were repeated in a nominally $\mathrm{Ca}^{2+}$ free extracellular solution. In these experiments the probability of finding $\mathrm{Ca}^{2+}$ cyt oscillations was reduced to that of untreated control cells by the addition of $5 \mathrm{mM}$ EGTA to the buffer (Fig.S1A). We further measured the mean fluorescence over a time window of $60 \mathrm{~min}$ to $120 \mathrm{~min}$ after Xray exposure. The value was significantly higher in cells treated with 5 Gy X-ray than that of the shamirradiated control group (Fig. S1B). In the presence of EGTA this value was greatly reduced and only 2 times higher than the control. The results of these experiments show that the absence of external $\mathrm{Ca}^{2+}$ abolishes radiation induced $\mathrm{Ca}^{2+}{ }_{\text {cyt }}$ oscillations but still allows some steady increase in $\mathrm{Ca}^{2+}$ cyt. This suggests a calcium influx via plasma membrane channels as the main trigger of $\mathrm{Ca}^{2+}$ cyt oscillations in irradiated cells. To further test this hypothesis, experiments were repeated in a buffer containing $\mathrm{Ca}^{2+}$ and \pm gadolinium $\left(\mathrm{Gd}^{3+}\right)$, a broad inhibitor of $\mathrm{Ca}^{2+}$ permeable channels in T cells $(19,20)$. In the presence of $5 \mu \mathrm{M}$ inhibitor the probability of radiation-induced $\mathrm{Ca}^{2+}{ }_{\text {cyt }}$ oscillations was reduced close to that in control cells (Fig. 1SA). Like EGTA Gd ${ }^{3+}$ did not fully prevent a radiation induced increase in the concentration of $\mathrm{Ca}^{2+}{ }_{\text {cyt }}$ above the sham-irradiated control (Fig. S1B). Together these data underscore the impact of radiation on $\mathrm{Ca}^{2+}{ }_{\text {cyt }}$ and the importance of $\mathrm{Ca}^{2+}$ influx for the radiation-induced signaling cascade, which leads to $\mathrm{Ca}^{2+}{ }_{\text {cyt }}$ oscillations.

\section{$\mathrm{Ca}^{2+}$ oscillations are mediated by STIM/Orai activation}

The major mechanism for $\mathrm{Ca}^{2+}$ entry into $\mathrm{T}$ cells is provided by the calcium induced calcium release (CICR) pathway (18). In this system the calcium level of endoplasmatic reticulum (ER) stores is monitored by the $\mathrm{ER} \mathrm{Ca}^{2+}$ sensors stromal interaction molecules (STIM). Upon store depletion STIMs aggregate and move to contact points with the plasma membrane (PM) where they interact and activate Orai channels (21). The active Orai1/STIM1 complex, which assembles to the CRAC channel (22), is necessary and sufficient to support SOCE. To test the involvement of this pathway in the radiation induced $\mathrm{T}$ cell stimulation we monitored the dynamic distribution of Orai1 and STIM1 in Jurkat cells and PBMCs. Cells were fixed for immunostaining 15, 30, 60 min after X-ray exposure or activating with TG. The representative images in Fig. 2A and Fig. S2 show the typical distribution of the two components in unstimulated cells. The Orai channel subunit is evenly distributed in the PM while the STIM protein generates a diffuse signal throughout the cell (Fig. 2, Fig. S2). After activating cells with $2 \mu \mathrm{M}$ TG, STIM proteins are translocated from the cytosol to the plasma membrane where they colocalize with the Orai proteins in distinct clusters.

The same distinct overlay of STIM and Orai in plasma membrane puncta emerged after exposing Jurkat cells to a dose of 5 Gy (Fig. 2A). Already 15 minutes after irradiation a maximum clustering of both proteins was observed in approximetly $50 \%$ of the cells analyzed before the signal gradually decreased (Fig. 2B). A similar transient clustering of STIM and Orai was evident with a 1.5 Gy X-ray exposure. In experiments with $0.5 \mathrm{~Gy}$ it was still possible to detect individual cells with a clear clustering of the two proteins (Fig. 2B, C). This confirms that low dose irradiation is able to elicit STIM/Orai aggregation; the numbers however were too low for a robust statistical analysis.

To confirm X-ray triggered aggregation of STIM and Orai we co-expressed STIM1::eYFP and Oari1::eCFP in Jurkat cells and measured protein/protein interactions by Förster resonance energy transfer (FRET). Data in Figs 2D,E show that the FRET signal is small in untreated control cells. It significantly increased in cells stimulated with $2 \mu \mathrm{M}$ TG or $25 \mu \mathrm{L} / \mathrm{mL}$ ImmunoCult human CD3/CD28/CD2 T cell 
activator (T-Ac). An even larger energy transfer was measured in cells exposed to a dose of $5 \mathrm{~Gy}$. Together these data confirm that STIM1 and Orail interact in response to X-ray exposure.

To estimate the time course for stimulus-induced STIM1/Orail clustering we measured in cells, which co-express both proteins, the Pearson correlation coefficient (PCC) for the yellow fluorescent STIM1 and the cyan fluorescent Orail after stimulation. The mean colocalization values in unstimulated cells were low ( $\mathrm{PCC}=0.3 \pm 0.08$ ) and did not change over time (Fig. 2C). After stimulation the PCC value increased rapidly in response to $\mathrm{TG}(\mathrm{PCC}=0.76 \pm 0.05)$ and slow after adding the activator $\mathrm{T}-\mathrm{Ac}(\mathrm{PCC}=0.79 \pm 0.04)$. The irradiation-induced increase in STIM1/Orail cluster formation progressed with the same slow kinetics of the activator to a maximal PCC value of $0.79 \pm 0.06$. The kinetics of changes in the PCC values could be fitted by a logistic equation:

$$
\mathrm{Y}=\mathrm{a} /\left(1+\mathrm{e}^{-\mathrm{k}^{*}(\mathrm{x}-\mathrm{xo})}\right)
$$

where $\mathrm{a}$ is the maximal PCC value, $\mathrm{k}$ the rate of increase and $\mathrm{x}_{0}$ the time of maximal increase.

In case of the 5 Gy exposure the maximal increase in co-localization was achieved $\sim 12$ min after stimulation (Fig. 2C). This value is in good agreement with the mean delay between X-ray stimulation and the onset of $\mathrm{Ca}^{2+}$ cyt oscillations as depicted in Fig. 1.

\section{The STIM/Orai activation pathway is also activated in naïve T-lymphocytes.}

Jurkat cells are a leukemic $\mathrm{T}$ cell line, which serves as a model system for uncovering the basic signaling events engaged in $\mathrm{T}$ cell activation (23). To test whether the irradiation triggered $\mathrm{Ca}^{2+}$ signaling cascade is also occurring in naïve T cells, we repeated the experiments in Fig. 2 with PBMCs. The images in Fig. $3 \mathrm{~A}$ indicate that the cytosol volume of non-stimulated lymphocytes is even smaller than that of Jurkat cells, which makes it more difficult to detect a translocation of STIM from the cytosol to the membrane resident Orai. A comparative analysis nonetheless shows that the Orai and STIM distribution remains uniform in unstimulated control cells while they exhibit a distinct clustering after stimulation with $2 \mu \mathrm{M}$ TG. The same clustering is also evident after exposing cells to 5 Gy (Fig. 3A). This underscores that the IR induced $\mathrm{Ca}^{2+}$ signaling cascade is a general response of resting $\mathrm{T}$ cells.

\section{$\mathrm{Ca}^{2+}$ signaling cascade results in a translocation of NFAT to the nucleus.}

$\mathrm{Ca}^{2+}$ entry via the SOCE pathway is the main source for activating the transcription factor isoforms of NFAT $(24,25)$. The stimulus-induced and $\mathrm{Ca}^{2+}$-dependent nuclear translocation of NFAT is instrumental for subsequent cytokine expression, proliferation and immune competence (26). Notably, the frequency of the X-ray induced $\mathrm{Ca}^{2+}$ oscillations in Jurkat cells (Fig. 1E) is typical for a signaling cascade, which elicits the activation of the NFAT pathway (27).

To test if the NFATc2 pathway is indeed activated by ionizing radiation we monitored nuclear translocation of endogenous NFAT labelled with Alexa (Alx)488. The representative images depicted in Fig. $3 \mathrm{~B}$ and $4 \mathrm{~A}$ show that the transcription factor is primarily located in the cytosol in unstimulated Jurkat cells (4A) and naïve T cells (3B). Stimulation with $2 \mu \mathrm{M}$ TG favors a translocation of NFAT into the nucleus in a population of cells tested (Fig. S1C). The similar nuclear translocation is induced in $72 \%$ of cells irradiated with 5 Gy(Fig. 4A,B, Fig. S1C).

Stimulus-induced translocation of the heterologous expressed GFP tagged NFAT can be monitored with live cell imaging by following its translocation dynamics in real-time (Fig. 4B). Again, analyses of NFAT accumulation in the nucleus highlights distinct response times to different stimuli. While TG stimulation causes a $50 \%$ nuclear translocation of NFAT (in $97 \%$ off all cells monitored) after $8 \pm 1.5$ min, it takes more than $21 \pm 6.5$ minutes for a comparable response in $89 \%$ of the cells stimulated with T-Ac and $70 \pm 5$ minutes for a 5 Gy irradiation $67 \%$ of all cells (Fig. 4C). The nuclear translocation of NFAT is causally related to the activation of CRAC channels for all three stimuli as treating cells with the CRAC channel inhibitor Synta abolishes the nuclear translocation of NFAT (Fig. 4C; Fig. S1C) in all cases.

Finally, to examine the consequence of CRAC channel activation on the physiological response of Jurkat cells we examined the effect of $10 \mu \mathrm{M}$ Synta on irradiation induced increase in cell diameter; since 
the morphological change is part of the radiation-induced immune response (14). The data depicted in Fig. 4D indicate that the cells have a uniform diameter under control conditions. Over $24 \mathrm{~h}$, this diameter is not changing neither in the presence of $10 \mu \mathrm{M}$ Synta nor in the absence of Synta. X-ray irradiation with three different doses $(0.5,1.5$ and $5 \mathrm{~Gy})$ caused a dose-dependent increase in cell diameter. This increase in cell size was largely abolished in the presence of the CRAC channel blocker (Fig. 3D). The results of these experiments suggest that the morphological change in Jurkat cells in response to X-ray stimulation is an endpoint of a signal transduction cascade, which involves SOCE via STIM1/Orail clustering with subsequent NFATc2 activation.

\section{Discussion}

Stimulus induced $\mathrm{Ca}^{2+}$ signaling cascades are a key event in the activation of $\mathrm{T}$ cells. Triggered by antigen binding to the $\mathrm{T}$ cell receptor (28), a downstream signaling cascade is initiated, which promotes the release of $\mathrm{Ca}^{2+}$ from internal stores and eventually the activation of SOCE. In this process $\mathrm{Ca}^{2+}$ enters the cytoplasm primarily via CRAC channels and generates $\mathrm{Ca}^{2+}$ cyt oscillations with distinct durations and amplitudes $(29,30)$. These dynamic changes in the concentration of the second messenger molecule are finally decoded by cytosolic $\mathrm{Ca}^{2+}$-dependent target enzymes including kinases, phosphatases and transcription factors such a NFAT (31-33). With this network of signaling steps T cells achieve precise control over essential lymphocyte functions such as cytokine production, proliferation, differentiation and antigen dependent cytotoxicity. In the present study, we indicate that the signaling cascades involving CRAC channel activation, SOCE-mediated $\mathrm{Ca}^{2+}{ }_{\text {cyt }}$ excursions and translocation of NFAT from the cytosol to the nucleus can be triggered in a population of $T$ cells by clinically relevant doses of ionizing irradiation. In this regard IR elicts comparable effects to a well-established activator of T cells or of TG. Further, the finding that a specific inhibitor of CRAC channels blocks the crucial translocation of NFAT and physiological responses of $\mathrm{T}$ cells irrespectively of the nature of stimulation underpins that they all employ SOCE as the major $\mathrm{Ca}^{2+}$ entry pathway. Other $\mathrm{Ca}^{2+}$ channels in $\mathrm{T}$ cells seem to play no primary role in IR-induced $\mathrm{Ca}^{2+}$ signaling.

It is well established that the form of $\mathrm{Ca}^{2+}$ cyt signals in $\mathrm{T}$ cells determines their cellular response (30, 34-36). Depending on the nature and the concentration of the stimuli the $\mathrm{Ca}^{2+}$ signal can either exhibit a sustained increase in $\mathrm{Ca}^{2+}{ }_{\text {cyt }}$ or periods of $\mathrm{Ca}^{2+}$ cyt-oscillations with different frequencies and amplitudes. This frequency and amplitude encoded signature of the $\mathrm{Ca}^{2+}$ cyt oscillations bears information on the subsequent differentiation of T cells; it can induce proliferation or cell death (37). Different amplitudes and frequencies also activate different transcription programs in populations of T cells (38). Scrutiny of IR -nduced $\mathrm{Ca}^{2+}$ oscillations show that they oscillate independent on the IR doses with frequencies of $2-4 \mathrm{mHz}$. This is in the frequency range of antigen-triggered oscillations in T cells which eventually result in an activation of NFAT $(24,27)$. Our finding that IR activates the NFAT pathway in T cells is in good agreement with the view that both stimuli trigger the same signaling pathways.

A peculiarity of IR triggered $\mathrm{Ca}^{2+}$ cyt oscillations is their high amplitude. They reach the same level as those induced by ionomycin suggesting that the peak amplitudes must exceed $\mathrm{Ca}^{2+}$ cyt concentrations of 1 $\mu \mathrm{M}$ e.g. the concentration at which the fluorescent sensor approaches saturation. The amplitudes of TGinduced excursions of $\mathrm{Ca}^{2+}$ cyt are well below this value suggesting that the response of T cells to IR is similar but not identical to a canonical antigen stimulation by $\mathrm{T}$ - Cell activator. This difference in the $\mathrm{Ca}^{2+}$ code may contribute to the findings that IR does not elicit the same degree of immune activation in T cells than the activator (14). This might bear consequences for the spectrum of physiological reactions, which are triggered by IR. Important to consider in this context is that a high $\mathrm{Ca}^{2+}$ load can induce cell death in lymphocytes. Together with the finding that the amplitude of the $\mathrm{Ca}^{2+}{ }_{\text {cyt }}$ oscillations increase with the IR, this might be a possible explanation for an induced death of T cells by high dose IR exposure (39).

An interesting finding in the present study is that IR induced $\mathrm{Ca}^{2+}$ cyt oscillations are not an immediate consequence of radiation exposure; they only occur with a delay of several minutes. This delay is well beyond the life-time of oxygen radicals including the long lived $\mathrm{H}_{2} \mathrm{O}_{2}(40,41)$ meaning that $\mathrm{Ca}^{2+}$ oscillations are not initiated by radiolysis of water or peroxidation/oxidation of lipids/proteins. This lack of an immediate impact of IR on $\mathrm{Ca}^{2+}{ }_{\text {cyt }}$ is consistent with previous high-resolution imaging experiments which showed that 
individual high energy ions had no immediate impact on the $\mathrm{Ca}^{2+}$ concentration along the track of the ions (42). The current data are not sufficient to explain the gap between IR exposure and the onset of $\mathrm{Ca}^{2+}{ }_{\text {cyt }}$ oscillations. Worth noting, however, is that typical $\mathrm{T}$ cell stimuli like phytohemagglutinin are not causing an immediate $\mathrm{Ca}^{2+}$ response (43); like in the case of IR it takes up to $5 \mathrm{~min}$ before $\mathrm{Ca}^{2+}$ cyt starts to oscillate. This parallel in the dynamics between antigen and IR-induced events is even more evident in the time course of STIM/Orai clustering, which shows about the same time course irrespectively of a stimulation by IR or by a $\mathrm{T}$ cell activator. If these parallels are a coincidence or based on a common mechanism like generation of inositoltrisphosphate $\left(\mathrm{IP}_{3}\right)$ and/or diazylglycerol (DAG) upstream of SOCE needs to be investigated.

Since immune cells like T cells, which are cycling in the blood, are inevitably exposed to IR during tumor therapy, it is important to understand their response to IR. The present study underpins a stimulating role of IR on STIM/Orai cluster formation and a subsequent activation of CRAC channels for SOCE not only in a $\mathrm{T}$ cell cancer cell line (Jurkat cells), but also in naïve peripheral blood lymphocytes. Tlymphocyte activation is in the forefront of anti-tumor cytotoxic effects and the regulation of an adaptive immune response in healthy and cancerous tissue $(44,45)$. Our findings thus have implications for the understanding of both, radiation associated toxicity in normal tissue and the efficacy of radiation therapy, especially if combined with checkpoint PD1 and PD-L1 inhibitors in current clinical practice. Further, STIM and Orai proteins are not restricted to T cells but also expressed in B-cells as well as in the phagocytes such as neutrophilic granulocytes, macrophages, and dendritic cells (46) where they regulate a multitude of cellular reactions (15). With a more general functional importance of these channel forming proteins in different types of immune cells we anticipate that IR activation of CRAC channels will even have a more global importance in the modulation of the immune responses following IR.

\section{Materials and Methods}

Cell culture: Jurkat cells (ACC 282) were purchased from the German Collection of Microorganisms and Cell Cultures (DSMZ, Braunschweig, Germany). They were grown in RPMI 1640 medium (Thermo Fischer Scientific Waltham, MA, USA), supplemented with 10\% heat inactivated fetal calf serum (FCS; PAA, Cölbe, Germany) and $50 \mathrm{U} / \mathrm{ml}$ penicillin plus $5 \mu \mathrm{g} / \mathrm{ml}$ streptomycin (Sigma-Aldrich, Munich, Germany). Peripheral blood mononuclear cell (PBMCs) were isolated from blood of healthy volunteers, using density-gradient centrifugation (Biocoll Separating Solution, Biochrom, Berlin, Germany) and maintained in RPMI 1640 Medium with $10 \%$ FCS, plus $50 \mathrm{U} / \mathrm{ml}$ penicillin and $5 \mu \mathrm{g} / \mathrm{ml}$ streptomycin prior to assays as described previously (14).

Cell irradiation and treatments: Cells were exposed to X-ray irradiation in $\mathrm{T}_{35}$ petri dishes using an Isovolt 160 Titan E source with a voltage of $90 \mathrm{kV}$ and $33.7 \mathrm{~mA}$ (GE Sensing \& Inspection Technologies, Alzenau, Germany), with a dose rate of $0.055 \mathrm{~Gy} / \mathrm{s}$. Ionomycin (\# ab120370, Abcam, Cambridge, UK), thapsigargin (TG) (Sigma-Aldrich, Taufkirchen, Germany) and the cell-permeable $\mathrm{Ca}^{2+}$ sensor Fluo-4 AM (Life Technologies, Carlsbad, CA, USA) were dissolved in DMSO and immediately added to external solution prior to experiments in a final concentration of $5 / 2 / 1 \mu \mathrm{M}$, respectively. To activate human $\mathrm{T}$ cells, ImmunoCult ${ }^{\mathrm{TM}}$ Human CD3/CD28/CD2 T cell activator (short T-Ac) (Stem cell Technologies, Vancouver, $\mathrm{BC}$, Canada) was added to the cell culture medium ( $25 \mu \mathrm{L}$ per $1 \mathrm{~mL}$ of cell suspension). The cell permeable organelle tracker Mito-Tracker Green FM (\#M7514) and ER-Tracker Red (\#E34250) and PM tracker CellMaskOrange (Thermo Fisher Scientifc) were used according to the manufacturer's recommendations. Cell nuclei were stained with Hoechst dye ( $5 \mu \mathrm{g} / \mathrm{ml}$, Thermo Fisher Scientifc) diluted in external microscopy buffer, for $10 \mathrm{~min}$ at $37^{\circ} \mathrm{C}$. Subsequently, cells were washed and resuspended in dye-free microscopy buffer. Determination of cell diameters: Jurkat cell diameters were measured with an EVE automatic cell counter (NanoEnTek, Seoul, South Korea) and corrected manually using EVE PC.LNK 1.0.3 software. Cell viability was determined by using trypan blue exclusion.

Confocal laser scanning microscopy: Confocal laser scanning microscopy was performed on a Leica TCS SP or SP5 II system (Leica microsystems, Mannheim, Germany) equipped with a $40 \times 1.30$ oil UV (HCX PL APO), a $63 \times 1.4$ oil UV (HCX PL APO lambda blue) or a $100 \times 1.44$ oil UV objective (HCX PL APO CS). The external buffer used for microscopy (MB) contained in mM: $140 \mathrm{NaCl}, 4 \mathrm{KCl}, 1 \mathrm{MgCl}_{2}, 5 \mathrm{Mannitol}$, 10 HEPES, $2 \mathrm{CaCl}_{2}$, pH 7.4 with osmolarity of 310 mosmol/L. Live cell imaging of changes in $\mathrm{Ca}^{2+}{ }_{\text {cyt }}$ and 
translocation of NFAT-GFP were performed as described in (14) and (47) respectively. STIM1-eYFP and ORAI-eCFP were transiently expressed as described in (21). To enable a gentle adhesion of the cells to the glass coverslips (Ø $25 \mathrm{~mm}$ ) they were prepared by cleaning in a plasma furnace (Zepto-B, Diener electronic $\mathrm{GmbH}$, Ebhausen, Germany) and coated with one layer of PBS/5\% BSA in a spincoater (PIN150, SPS Europe Spincoating, Putten, Netherlands) and a second layer of 0.01\% poly-L-lysine (molecular weight 75 $150 \mathrm{kDa})$.

For monitoring the $\mathrm{Ca}^{2+}$ cyt Jurkats were loaded with the cell permeable $\mathrm{Ca}^{2+}$ sensor Fluo-4 (Life Technologies) for $30 \mathrm{~min}$ in microscopy buffer in a final concentration of $1 \mu \mathrm{M}$. The calcium dye was subsequently removed by washing cells with dye-free buffer. Calcium signals were recorded in a time interval of $5 \mathrm{~s}$ for 60-240 min in total with an image resolution of $1024 \times 1024$ pixel and a scan speed of $400 \mathrm{~Hz}$. Transfection of the immune cells for transiently expressed proteins was accomplished with lipofectamin 2000 (Thermo Fisher Scientific) according to the manufacturer's instructions. Live-cell analysis of heterologous expressed NFATc2-GFP and STIM1-eYFP/Orail-eCFP localization was performed using the CLSM mentioned above. The microscopy settings were as follows - an image resolution of 1024 x 1024 pixel, scan speed of $200 \mathrm{~Hz}$, time interval of $60 \mathrm{~s}$ for $30-100 \mathrm{~min}$ in total.

FRET Analysis: FRET experiments with Jurkat cells, transiently expressing STIM1-eYFP/Orai1-eCFP, were examined with a Leica TCS SP5 II confocal microscope. Filter were set with CFP (458 Ex/460-490 Em), YFP (514 Ex/530-550 Em), and FRETraw (458 Ex/530-550 Em). Live-cell images were obtained every 30 $\mathrm{s}$ at room temperature with a $100 \times 1.44$ oil UV objective (HCX PL APO CS) for a time period of $30 \mathrm{~min}$. Three-channel corrected FRET was calculated based on the following equation: FRETc $=$ Fraw $\mathrm{Fd} / \mathrm{Dd} \cdot \mathrm{FCFP}-\mathrm{Fa} / \mathrm{Da} \cdot \mathrm{FYFP}$, where FRETc represents the corrected total amount of energy transfer; Fd/Dd is the measured bleed-through of eCFP via YFP filter (0.473); Fa/Da represents measured bleed through of YFP through CFP filter (0.049). To reduce variations caused by differences in expression levels of CFP, the FRETc values were normalized to value of donor fluorescence (FCFP). To minimize the effect of variations of YFP expression levels on FCFP-normalized FRET signals (FRETN) and to show the relative changes as compared with resting levels, figures are shown as $\triangle$ FRETN/FRETNrest.

Immunofluorescence staining: Jurkat cells were fixed on coated glass coverslips at 15, 30, 45, 60 or 90 min post treatment using $4 \%$ paraformaldehyde and next stained with primary antibodies for STIM1 (\#PA146217, Thermo Fisher Scientifc), Orail (\#NBP1-75523, Novus Biologicals, Waltham, MA, USA) and NFATc2 (\#MA1-025, Thermo Fisher Scientific). Antibodies were applied at a 1:200 dilution in PBS and coverslips were shaken over night at $4^{\circ} \mathrm{C}$. Next, cells were incubated with anti-rabbit IgG Alexa488 secondary antibody (\# A32731, Thermo Fisher Scientifc), anti-mouse IgG Alexa488 secondary antibody (\# A32723, Thermo Fisher Scientifc) or anti-mouse IgG Alexa647 secondary antibody (\# A32728, Thermo Fisher Scientifc).

Statistics: Data are expressed as mean \pm standard deviation (SD) or standard error (SE) of $\geq$ three independent experiments; number of biological replicates (n) or independent experiments $(\mathrm{N})$ are given in the text. Significance was estimated with Student's t-test. P values $<0.05(*),<0.01\left({ }^{* *}\right)$ and $<0.001(* * *)$ are indicated in the figures.

\section{Supplementary Materials}

Figure S1. Radiation-induced $\mathrm{Ca}^{2+}{ }_{\text {cyt }}$ oscillations and nuclear NFAT translocation are triggered by X-ray and abolished after inhibition of $\mathrm{Ca}^{2+}$ influx.

Figure S2. In resting Jurkat cells STIM1 and Orai1 are located in the ER and PM respectively

\section{References and Notes}

(1) Delaney G, Jacob S, Featherstone C, Barton M (2005) The role of radiotherapy in cancer treatment: Estimating optimal utilization from a review of evidence-based clinical guidelines. Cancer 104:11291137.

(2) Seegenschmiedt, MH Micke, O Muecke R (2015) Radiotherapy for non-malignant disorders: state of the art and update of the evidence-based practice guidelines. Br J Radiol 88: 20150080 
(3) Rödel F, Frey B, Mandam K, Hildebarndt G, Hehlgans S, Keilholz L, Seegenschmiedt MH, Gaipl US, Rödel C (2012) Immunomodulatory properties and molecular effects in inflammatory diseases of lowdose X-irradiation. Front Oncol 2:120.

(4) Bauer M, Goldstein M, Christmann M, Becker H, Heylmann D, Kaina B (2011) Human monocytes are severely impaired in base and DNA double-strand break repair that renders them vulnerable to oxidative stress. Proc. Natl. Acad. Sci. U.S.A. 108:21105-21110.

(5) Heylmann D, Rödel F, Kindler T, Kaina B (2014) Radiation Sensitivity of Human and Murine Peripheral Blood Lymphocytes, Stem and Progenitor Cells. Biochim Biophys Acta 1846:121-129.

(6) Stoecklein VM, Osuka A, Ishikawa S, Lederer RM, Wanke-Jellinek L, Lederer JA (2015) Radiation Exposure Induces Inflammasome Pathway Activation in Immune Cells. J Immunol 194: 1178-1189.

(7) Donnelly EH, Nemhauser JB, Smith JM, Kazzi ZN, Farfán EB, Chang AS, Naeem F (2010) Acute radiation syndrome: assessment and management. South Med J 103:541-546

(8) Goans RE, Waselenko JK (2005) Medical management of radiological casualties. Health Phys. 89:505512.

(9) Di Maggio FM, Minafra L, Forte GI, Cammarata FP, Lio D, Messa C, Gilardi MC, Bravata V (2015) Portrait of inflammatory response to ionizing radiation treatment. J Inflamm 12:14.

(10) Lumniczky K, Szytmári T, Sáfrány G (2017) Ionizing radiation-induced immune and inflammatory reactions in the brain. Front Immunol 8: 517

(11) Frey, Rückert M, Deloch L, Rühle PF, Derer A, Fietkau R, Gaipl US (2017) Immunomodulation by ionizing radiation-impact for design of radio-immunotherapies and for treatment of inflammatory diseases. Immunol Rev 280:231-248

(12) Sharabi AB, Lim M, DeWeese TL, Drake CG (2015) Radiation and checkpoint blockade immunotherapy: radiosensitisation and potential mechanisms of synergy. Lancet Oncol. 16:e498-e509.

(13) Cuttler JM (2020) Application of low doses of ionizing radiation in medical therapies. Dose Response 18:1559325819895739

(14) Voos P, Fuck S, Weipert F, Babel L, Tandl D, Meckel T, Hehlgans S, Fournier C, Moroni A, Rödel $\mathrm{F}$, Thiel G (2018) Ionizing radiation induces morphological changes and immunological modulation of Jurkat cells. Front Immunol 9:922.

(15) Vaeth M, Feske S (2018) NFAT control of immune function: new frontiers for an abiding trooper. F1000Res. 7:260

(16) Dustin LB (2000) Ratiometric analysis of calcium mobilization. Clinical and Applied Immunol Reviews 1:5-15.

(17) Gee KR, Brown KA, Chen W-NU, Bishop-Stewart J, Gray D (2000) Chemical and physiological characterization of fluo-4 $\mathrm{Ca}^{2+}$-indicator dyes. Cell Calcium, 27:97-106.

(18) Trebak M, Kinet JP (2019) Calcium signalling in T cells. Nat Rev Immunol. 19:154-169.

(19) Biagi, BA, Enyeart JJ (1990) Gadolinium blocks low- and high-threshold calcium currents in pituitary cells. American Journal of Physiology - Cell Physiology 259:28-30.

(20) Adding LC, Bannenberg GL, Gustafsson, LE (2001) Basic experimental studies and clinical aspects of gadolinium salts and chelates. Cardiovascular Drug Reviews, 19:41-56

(21) Zheng S, Ma G, He L, Zhang T, Li J, Yuan X, et al. (2018) Identification of molecular determinants that govern distinct STIM2 activation dynamics. PLoS Biol 16: e2006898.

(22) Gudlur A, Hogan P.G. (2017) The STIM-Orai Pathway: Orai, the Pore-Forming Subunit of the CRAC Channel. In: Groschner K., Graier W., Romanin C. (eds) Store-Operated $\mathrm{Ca}^{2+}$ Entry (SOCE) Pathways. Advances in Experimental Medicine and Biology, vol 993. Springer, Cham.

(23) Abraham R, Weiss A (2004) Jurkat T cells and development of the T-cell receptor signalling paradigm. Nat Rev Immunol 4:301-308.

(24) Dolmetsch, RE, Xu K, Lewis RS (1998) Calcium oscillations increase the efficiency and specificity of gene expression. Nature 392:933-936.

(25) Tomida T, Hirose K, Takizawa A, Shibasaki F, Iino M (2003) NFAT functions as a working memory of $\mathrm{Ca}^{2+}$ signals in decoding $\mathrm{Ca}^{2+}$ oscillation. EMBO J 22: 3825-3832.

(26) Crabtree GR (2002) NFAT Signaling: Choreographing the social lives of cells. Cell 109: 67-79. 
(27) Smedler E (2014) Frequency decoding of calcium oscillations. Biochem Biophys Acta 1840: 964969.

(28) Bryceson YT, March ME, Ljunggren H-G, Long EO (2006) Synergy among receptors on resting NK cells for the activation of natural cytotoxicity and cytokine secretion. Blood 107:159-166.

(29) Salazar C (2008) Decoding of Calcium Oscillations by Phosphorylation Cycles: Analytic Results. Biophys J 94:1203-1215.

(30) Parekh AB (2011) Decoding cytosolic $\mathrm{Ca}^{2+}$ oscillations. Trends in Biochem Sci 36: 78-87.

(31) Cifuentes ME, Honkanen L, Rebecchi MJ (1993) Proteolytic fragments of phosphoinositidespecific phospholipase C- $\delta 1$. Catalytic and membrane binding properties. J Biol Chem 268: 1158611593.

(32) Kohout SC, Corbalán-García S, Torrecillas, A, Goméz-Fernandéz JC, Falke JJ (1994) C2 domains of protein kinase $\mathrm{C}$ isoforms $\alpha, \beta$, and $\gamma$ : Activation parameters and calcium stoichiometries of the membrane-bound state. Biochemistry, 41:11411-11424.

(33) Stemmer PM, Klee CB (1994) Dual Calcium Ion Regulation of Calcineurin by Calmodulin and Calcineurin B. Biochemistry, 33: 6859-6866.

(34) Bootmam MD, Berridge MJ (1995) The elemental principles of calcium signaling. Cell 83: 675678.

(35) Cahalan MD (2009) STIMulating store-operated $\mathrm{Ca}^{2+}$ entry. Nat Cell Biol 11:669-677.

(36) Song S. (2012) Irregular $\mathrm{Ca}^{2+}$ Oscillations Regulate Transcription via Cumulative Spike Duration and Spike Amplitude. J Biol Chem 287:40246-40255.

(37) Orrenius S, Zhivotovsky B, Nicotera P (2003) Regulation of cell death: The calcium-apoptosis link. Nature Rev Mol Cell Biol 4:552-565

(38) Dolmetsch R, Lewis RL, Goodnow CC, Healy JI (1097) Differential activation of transcription factors induced by $\mathrm{Ca}^{2+}$ response amplitude and duration. Nature 386:855-858

(39) Schaue D, McBride WH (2019) T lymphocytes and normal tissue responses to radiation. Front Oncol. 2:119.

(40) Sies H (2017) Hydrogen peroxide as a central redox signaling molecule in physiological oxidative stress: Oxidative eustress. Redox Biology, 11: 613-619.

(41) Mikkelsen RB, Wardman P (2003) Biological chemistry of reactive oxygen and nitrogen and radiation-induced signal transduction mechanisms. Oncogene 22:5734-5754.

(42) Du G, Fischer BE, Voss KO, Becker G, Taucher-Scholz G, Kraft G, Thiel G (2008) The absence of early calcium response to heavy ion irradiation in mammalian cells. Rad Res. 170: 316-326.

(43) Lewis RL, Cahalan MD (1989) Mitogen-induced oscillations of cytosolic $\mathrm{Ca}^{2+}$ and transmembrane $\mathrm{Ca}^{2+}$ current in human leukemic T cells. Cell 1: 99-112.

(44) Demaria S, Golden EB, Formenti SC. (2015) Role of Local Radiation Therapy in Cancer Immunotherapy. JAMA Oncol. 1:1325-1332

(45) Schaue D, McBride WH. (2012) T lymphocytes and normal tissue responses to radiation. Front Oncol. 2:119

(46) Demaurex N, Nunes P (2016) The role of STIM and ORAI proteins in phagocytic immune cells. Am J Physiol Cell. 310: c496-c508.

(47) Kehlenbach RH, Dickmanns A, Gerace L (1998) Nucleocytoplasmic Shuttling Factors Including Ran and CRM1 Mediate Nuclear Export of NFAT In Vitro. JCB. 141: 863-874

\section{Acknowledgments}

This work was supported in part by the German Research Foundation (DFG: Graduate school 1657) and by the German Federal Ministry of Education and Research (BMBF, grants no. 02NUK050A, 02NUK050C and 02NUK050D, GREWISalpha). We thank Donald Gill (Pennsylvania State University) and Ralph Kehlenbach (University of Göttingen) for providing STIM1-eYFP/Orail-eCFP and NFAT-GFP plasmids, respectively. Special thanks to Christine Gibhardt (University of Göttingen) for helpful suggestions. 


\section{Author contributions:}

Experiments, DT, TSp, SF, TS, SH and BJ; data analysis, DT, TS, GT; conceptualization, CF, FR, BR, AM, and GT; writing original draft preparation, DT, SF, BR, AM, GT; writing review and editing, FR, CF, GT; funding acquisition, CF, FR, GT. 
A
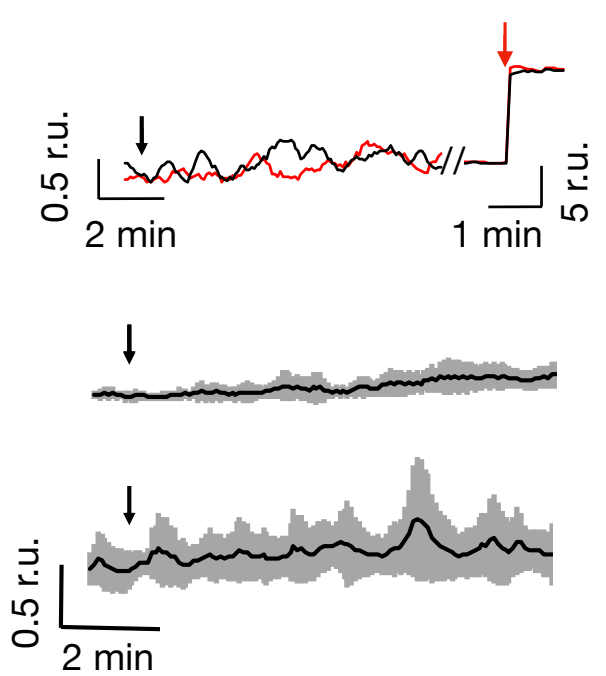

B
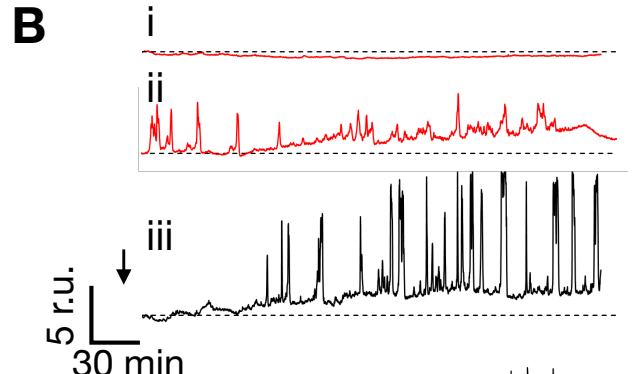

C

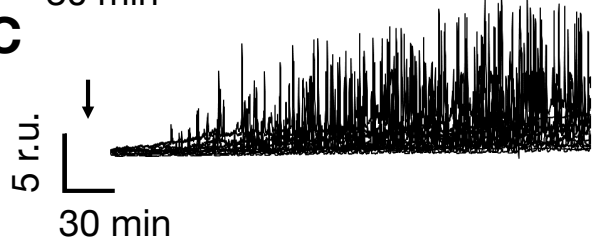

D

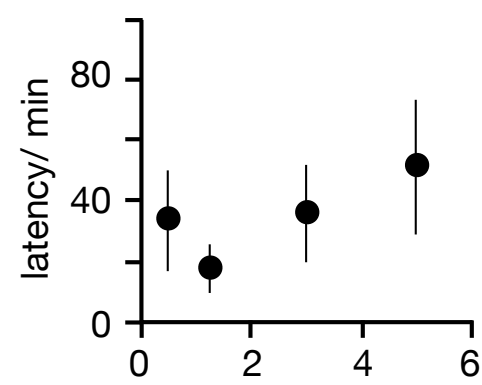

E

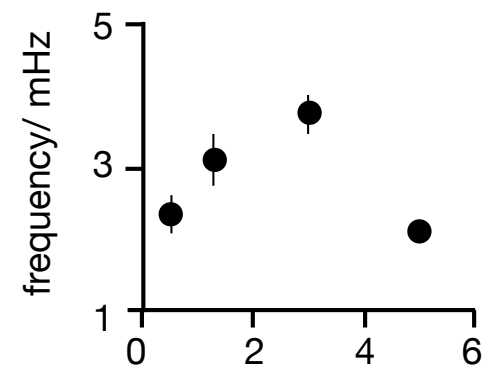

$\mathbf{F}$

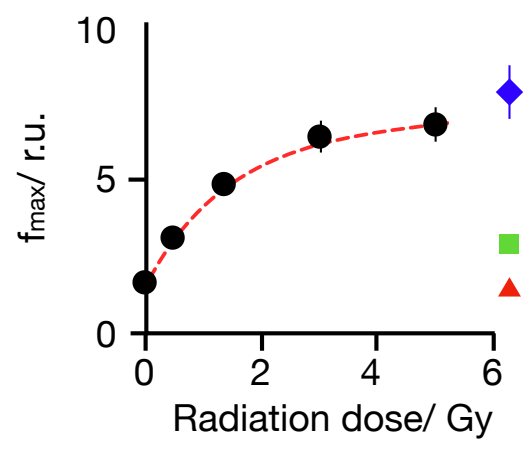

Figure 1. Ionizing irradiation elicits delayed $\mathrm{Ca}^{2+}{ }_{\text {cyt }}$ oscillations with distinct frequencies and amplitudes. (A) Representative Fluo-4 signals as measured as changes in cytosolic $\mathrm{Ca}^{2+}$ cyt in Jurkat cells (Top). Fluorescence was recorded in real-time before and after irradiation with $1 \mathrm{~Gy}$ (red) and 10 Gy (black) at times indicated by black arrows. The same cells responded with a maximal fluorescence increase after addition of $1 \mu \mathrm{M}$ ionomycin (red arrow). Mean Fluo-4 intensity from cells irradiated at arrow with $1 \mathrm{~Gy}$ (middle) or $10 \mathrm{~Gy}$ (bottom) X-ray. Data are means $\pm \mathrm{SD}$ (grey) from 15 cells for each dose. (B) Representative long-term measurements of Fluo-4 intensity in Jurkat cells of which two were non irradiated (i. ii, control, red) and the other exposed to a dose of $5 \mathrm{~Gy}$ Xray (iii, black). Time of X-ray exposure is indicated by black arrows. While control cells maintain a stable Fluo-4 signal (i) or reveal irregular oscillations (ii) the irradiated cell starts to oscillate after a lag time (iii). (C) Overlay of Fluo-4 signal from 15 individual cells after irradiation with 5 Gy; Time of X-ray exposure is indicated by black arrows.Latency time between onset of $\mathrm{Ca}^{2+}{ }_{\text {cyt }}$ oscillations after irradiation (D) oscillation frequency (E) and maximal amplitude of oscillation as a function of irradiation dose (F). Data are mean values $\pm \mathrm{SD}$ from $>25$ cells per dose. All $\mathrm{Ca}^{2+}$ cyt measurements in response to X-irradiation were performed in buffer containing $2 \mathrm{mM} \mathrm{Ca}^{2+}$. The colored symbols show the maximal level of Fluo-4 fluorescence intensity obtained in the same buffer by depleting $\mathrm{Ca}^{2+}$ stores with $2 \mu \mathrm{M}$ thapsigargin (blue triangle) or by adding $1 \mu \mathrm{M}$ ionomycin (green square); red triangles report Fluo-4 intensity elicited by $5 \mathrm{~Gy}$ in $\mathrm{Ca}^{2+}$ free external buffer. 


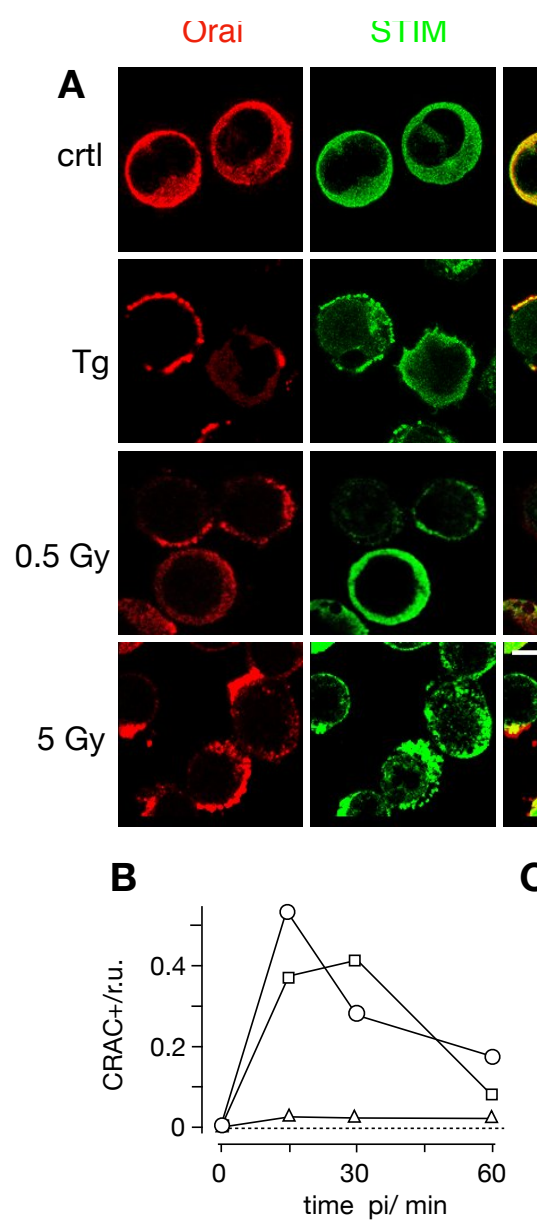

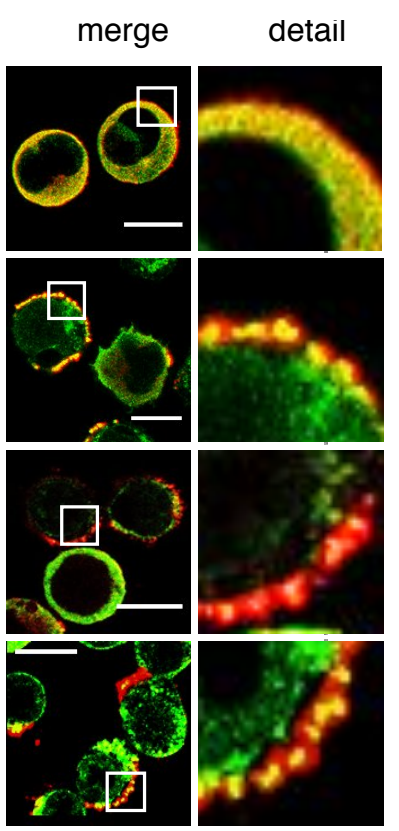

C

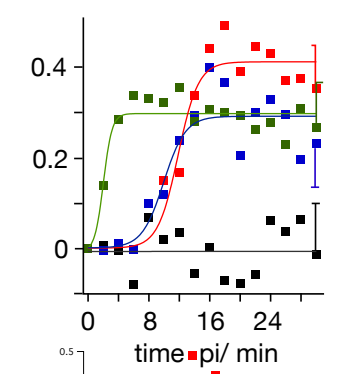

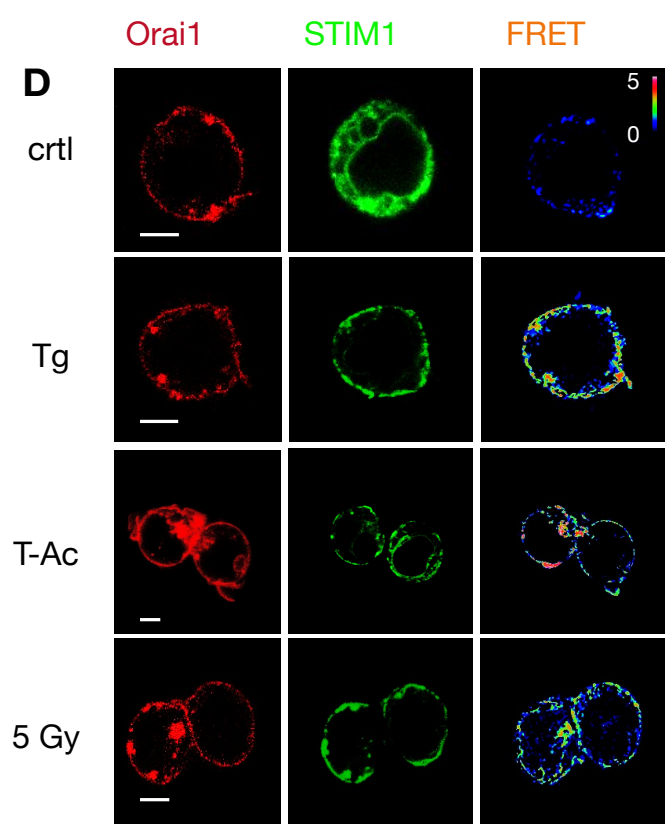

$\mathbf{E}$

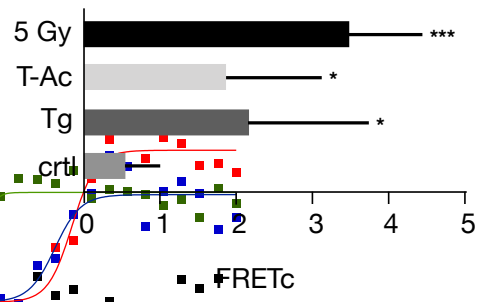

Figure 2. Ionizing radiation triggers $\mathrm{Ca}^{2+}$ regulated STIM1/Orai1 CRAC channel formation (A) Distribution of endogenous STIM1 (green $1^{\text {st }}$ column) and Orail (red $2^{\text {nd }}$ column) in Jurkat cells immunostained with Alexa (Alx)488 and Alx647, respectively. An overlay of green and red channels with magnification of indicated area are shown in $3^{\text {rd }}$ and $4^{\text {th }}$ columns. Fixed cells were obtained from untreated cells (top row), cell treated for 15 min with $2 \mu \mathrm{M}$ thapsigargin ( $2^{\text {nd }}$ row) or from cells $15 \mathrm{~min}$ after X-ray exposure to $0.5 \mathrm{~Gy}$ (third row) or $5 \mathrm{~Gy}$ (bottom row). (B) Frequency of Jurkat cells with positive clustering of STIM/Orail after store-depletion by irradiation with 0.5 Gy X-ray (triangles), $1.5 \mathrm{~Gy}$ (squares) or $5 \mathrm{~Gy}$ (circles). For each condition $\geq 282$ cells were analyzed. (C) Pearson correlation coefficient (PC) for colocalization of STIM1-eYFP and Orai1eCFP. Data obtained from confocal live cell real-time acquisition of Jurkat cells heterologously expressing the two proteins. After normalizing the start value of all treatments, the PC value remains at the same level in control cells (black) but increases with different kinetics in cells stimulated with $2 \mu \mathrm{M}$ thapsigargin (green), $25 \mu \mathrm{L} / \mathrm{mL}$ activator (blue) or $5 \mathrm{~Gy}$ (red) X-rays. The data were fitted with logistic equation (eqn 1, solid lines) yielding the following times for maximal increase in STIM1/Orai colocalization: 2 min TG, 10 min T-Ac, 12 min X-ray. Data are mean values $\pm \mathrm{SD}$ from $\geq 5$ independent experiments. The mean SD of all data points is shown on the last data points. All scale bars $10 \mu \mathrm{m}$. (D) Representative confocal images of same cells with fluorescent donor molecule Orail-eCFP (red, $1^{\text {st }}$ column), acceptor molecule STIM1-eYFP (green, $2^{\text {nd }}$ column) and heat maps of the resulting FRET signals ( $3^{\text {rd }}$ column). Images are from untreated cells (control), or cells incubated with $2 \mu \mathrm{M}$ thapsigargin, $25 \mu \mathrm{L} / \mathrm{mL}$ activator (T-Ac) or irradiated with $5 \mathrm{~Gy}$. Cells were fixed $15 \mathrm{~min}$ after start of treatment. All three treatments generate a visible FRET-signal in the plasma membrane. Scale bars, $10 \mu \mathrm{m}$. (E) Mean FRET signal ( \pm SD, $n \geq 5$ cells) from plasma membrane of cells treated as in D: control cells (crtl), $5 \mathrm{~min}$ in $2 \mu \mathrm{M} \mathrm{Tg}, 15 \mathrm{~min}$ in $25 \mu \mathrm{l} / \mathrm{ml} \mathrm{T}$-Ac or 20 min post irradiation with $5 \mathrm{~Gy}$. The FRET signal from treated cells is significantly higher than that of the control value $(* \mathrm{P}<0.05, * * * \mathrm{P}<0.001$ from Student's-test) 

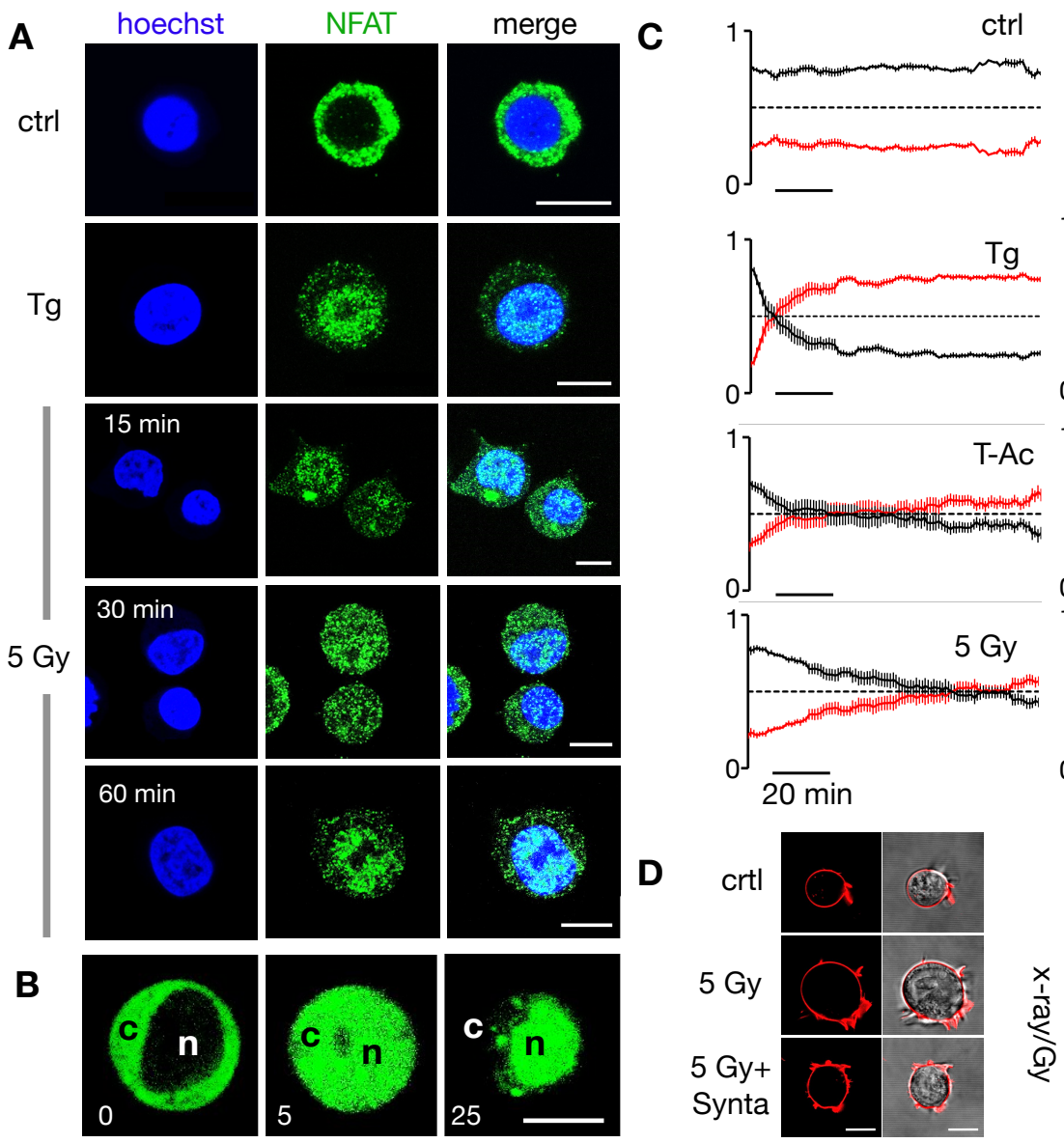

$+10 \mu \mathrm{M}$ Synta
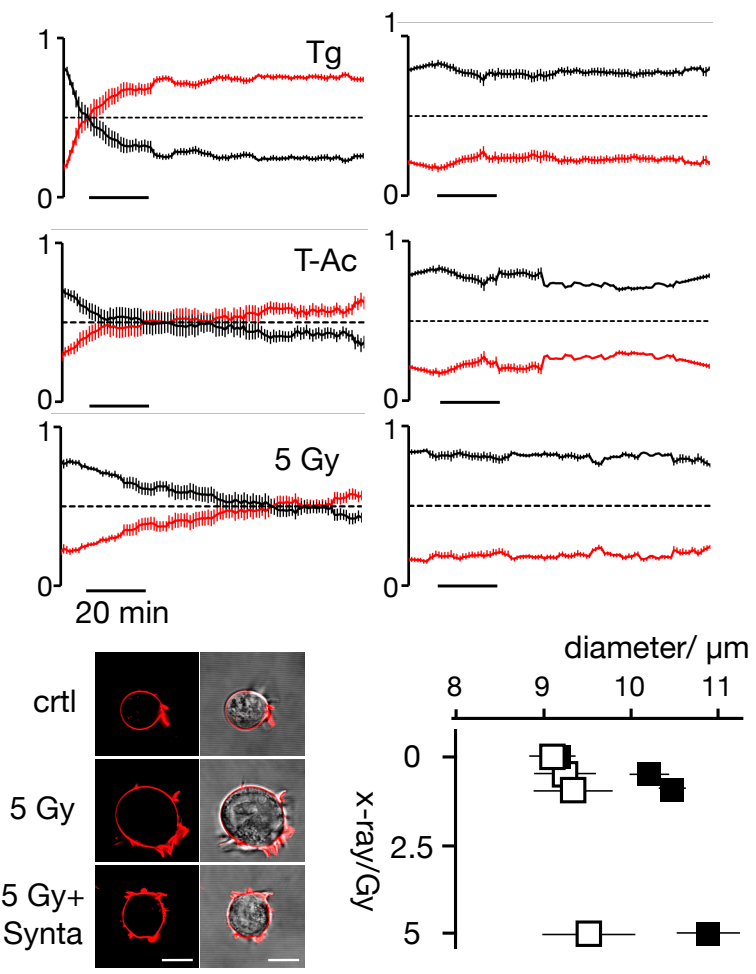

Figure 3. Calcium-dependent SOCE/NFAT pathway is activated by IR in naïve T-lymphocytes (A) Distribution auf endogenous STIM1 (green, left column) and Orai1 (red, $2^{\text {nd }}$ column) in fixed PBMCs immune-stained with Alx488 and Alx647 respectively. A merger of the two channels is shown in $3^{\text {rd }}$ column with a higher magnification of the marked area in the right column. In control cells also the nucleus is additionally stained with Hoechst dye. Cells were fixed (control cells, top) 15 min after treatment with $2 \mu \mathrm{M}$ thapsigargin (Top) or 15 min after exposing cells to either doses of 0.5 Gy or 5 Gy. (B) Confocal images of endogenous NFATc2 (green) stained with Alx488 (left column) and Hoechst DNA dye $\left(2^{\text {nd }}\right)$ in PBMC. Overlay of both columns is shown in the third row. Cells were fixed immediately (control, top), 15 min after $2 \mu \mathrm{M}$ thapsigargin $\mathrm{Ca}^{2+}$ store depletion (middle) or $60 \mathrm{~min}$ after X-ray exposure with $5 \mathrm{~Gy}$ (Bottom). All scale bars, $10 \mu \mathrm{m}$. 

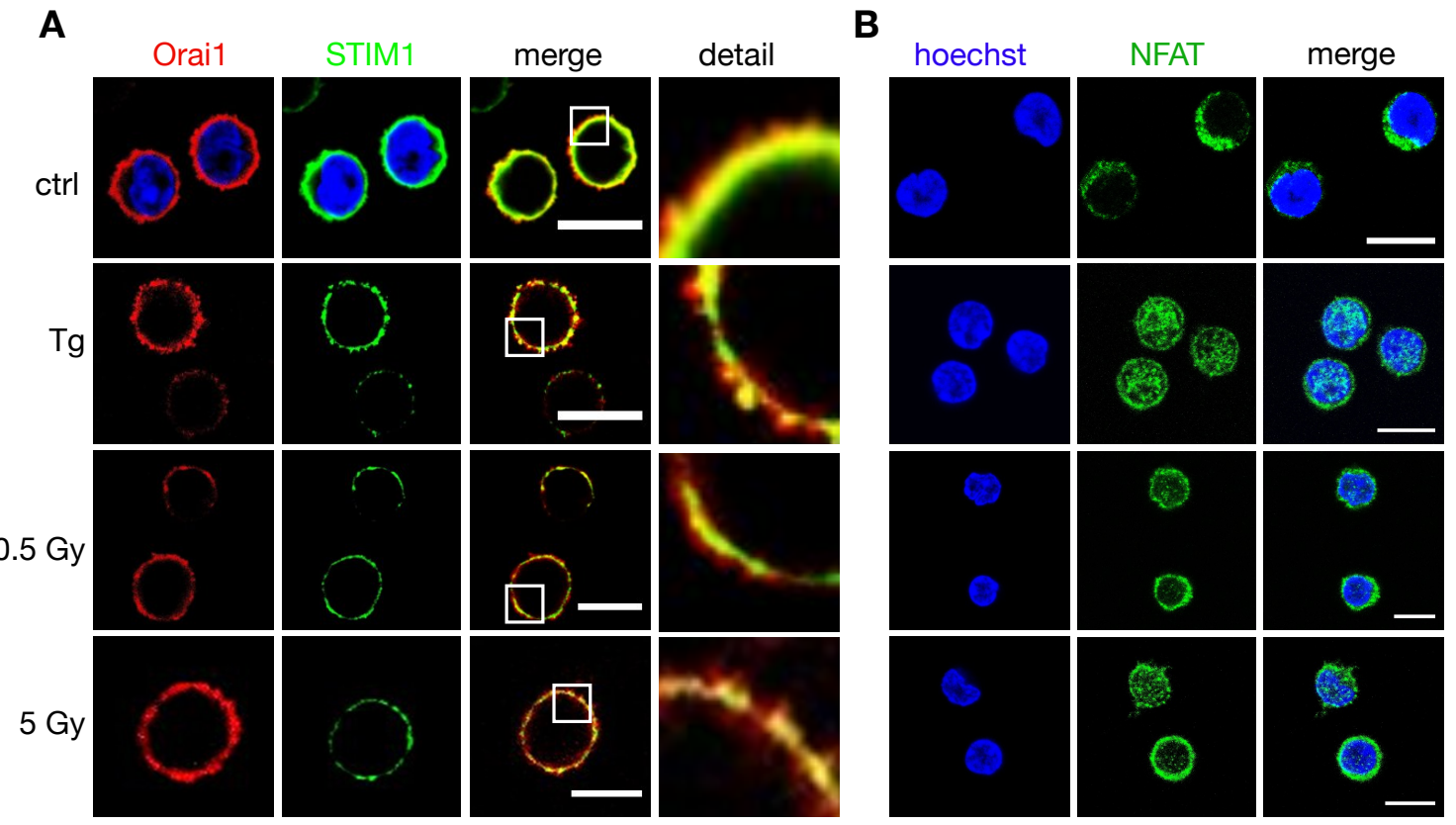

Figure 4. Nuclear translocation of $\mathrm{Ca}^{2+}$ - dependent NFAT in Jurkat cells

(A) Confocal images of endogenous NFATc2 (green) immunostained with Alx488 (green, left row) and Hoechst DNA dye (blue, $2^{\text {nd }}$ row) in Jurkat cells. The third row shows a merge of blue and green channels. Cells were fixed immediately in control cells (Top), 15 min after $2 \mu \mathrm{M}$ thapsigargin $\mathrm{Ca}^{2+}$ store depletion (second panel) or 30, 60 min after X-ray exposure with 5 Gy (two bottom panels).

(B) Life cell imaging of nuclear import of transiently expressed NFATc2-GFP from cytosol (c) to nucleus (n) in Jurkat cells after stimulation with $2 \mu \mathrm{M}$ thapsigargin.

(C) Kinetic analysis of NFATc2-GFP nuclear import reactions (nucleus red, cytosol black) from confocal imaging of Jurkat cells in control condition (crtl), in $2 \mu \mathrm{M}$ thapsigargin (Tg) in $25 \mu \mathrm{L} / \mathrm{mL}$ activator (T-Ac), or after a 5 Gy exposure (5 Gy). Data were obtained without (left) and with $10 \mu \mathrm{M}$ CRAC channel inhibitor Synta (right). Each time course diagram is the mean \pm S.E. of $\geq 12$ individually measured cells.

(D) Jurkat cells exhibit a dose-dependent increase in diameter $24 \mathrm{~h}$ after irradiation, which can be abolished by $10 \mu \mathrm{M}$ Synta. Representative images (left, confocal image of fluorescent stained plasma membrane (red) and overlay of fluorescent and bright field image) of individual Jurkat cells. Images were taken 24 after start of experiment with an untreated cell (ctrl), a cell exposed to Gy Xray without (5Gy) and with $10 \mu \mathrm{M}$ Synta (5Gy+Synta). Mean diameters \pm SD of $>300$ cells per treatment $24 \mathrm{~h}$ after exposed to X-ray between 0 and $5 \mathrm{~Gy}$ in the absence (black) or presence (open) $10 \mu \mathrm{M}$ Synta. The diameters \pm Synta are significantly different $\left({ }^{*} \mathrm{P}<0.05,{ }^{* *} \mathrm{P}<0.01,{ }^{* * *} \mathrm{P}<0.001\right.$ from student t-test). All scale bars, $10 \mu \mathrm{m}$. 